\title{
Potencial Termodinámico de un Refrigerador Intermitente por Adsorción bajo las Condiciones Climáticas de Riohacha, en Colombia
}

\author{
Aduar J. Camargo, José G. Quintero, Luis A. Avila y Gail A. Gutierrez* \\ Universidad de La Guajira, Fac. de Ingeniería, Programa de Ing. Mecánica, La Guajira-Colombia. \\ (e-mail: ajcamargo@uniguajira.edu.co; jgquintero@uniguajira.edu.co; lavilal@uniguajira.edu.co; \\ galbeirog@uniguajira.edu.co
}

* Autor a quien debe ser dirigida la correspondencia

Recibido Oct. 30, 2018; Aceptado Dic. 14, 2018; Versión final Abr. 9, 2019, Publicado Oct. 2019

\begin{abstract}
Resumen
La simulación computacional se usó para determinar el potencial que tiene el distrito turístico de Riohacha (Colombia) en la implementación de la refrigeración solar por adsorción a las condiciones climáticas locales (temperaturas ambientes e irradiación global). La termodinámica del ciclo es definida en el marco de la primera ley, usando calores totales, teniendo como parámetros de salida el coeficiente de funcionamiento (COP), el efecto de enfriamiento y la irradiación mínima para producir hielo. En la implementación, el algoritmo tuvo en cuenta la ecuación de Dubinin-Astakhove junto con los parámetros propios de la pareja de adsorción, la cual rige la adsorción en sólidos microporosos y se incluyó la línea de saturación del metanol. En los resultados, se obtuvieron gráficos de sensibilidad donde se reflejan el comportamiento del ciclo con respecto a la temperatura máxima, las temperaturas promedio ambiente, el COP, la irradiación mínima y la irradiación local durante diferentes días típicos del año. Los resultados sugirieron los meses del año con mejores potenciales para producir hielo según la temperatura máxima del ciclo.
\end{abstract}

\section{Thermodynamic Potential of an Intermittent Adsorption Refrigerator under the Climatic Conditions of Riohacha, in Colombia}

\begin{abstract}
Computer simulation was used to determine the potential of the Riohacha tourist district (Colombia), in the implementation of solar cooling by adsorption under local climatic conditions (ambient temperatures and global irradiation). The thermodynamics of the cycle are defined in the framework of the first law, using total heats, having as output parameter, the coefficient of performance (COP), the cooling effect and the minimum irradiation to produce ice. In the implementation, the algorithm took into account the Dubinin-Astakhove equation together with the parameters of the adsorption pair, which governs the adsorption in microporous solids and included the methanol saturation line. In the results, sensitivity graphs were obtained where the cycle behavior is reflected with respect to the maximum temperature, the average ambient temperatures, the COP, the minimum irradiation and the local irradiation during different typical days of the year. The results suggested the months of the year with the best potential to produce ice according to the maximum temperature of the cycle
\end{abstract}




\section{INTRODUCCIÓN}

El distrito de Riohacha (Departamento de La Guajira) se ubica sobre el mar caribe colombiano, coordenadas geográficas en $\left(11.5^{\circ} \mathrm{N}, 72.9^{\circ} \mathrm{O}, 3 \mathrm{~m}\right)$ y soleado casi todo el año. Según el atlas climatológico de Colombia("Atlas de Radiación Solar, Ultravioleta y Ozono de Colombia," n.d.), Riohacha cuenta con 8.3 horas de sol al día e irradiación global promedio entre 2000- $2200 \mathrm{kWh} / \mathrm{m} 2 / \mathrm{año}$ o 5.0-5.5 kWh/m2/día, convirtiéndose en una ventaja comparativa para las aplicaciones con energías no convencionales, una de tales aplicaciones versa sobre el refrigerador solar por adsorción física; una máquina térmica impulsada solo por calor de baja calidad, flexible para operar a pequeña, mediana y gran escala, casi libre de mantenimiento y ambientalmente amable (no usa CFC, HFC, etc.); especialmente apropiada para población vulnerable (Santori et al., 2014). Físicamente el refrigerador funciona con una cantidad másica de un adsorbente (carbón activado, CA) un adsorbato (refrigerante) capaz de ser adsorbido por el adsorbente, un colector solar-generador que contiene ambos materiales en estrecho contacto y dos intercambiadores de calor: uno actuando como condensador, enfriado por aire y otro como evaporador (Pons y Guilleminot, 1986). Esta máquina es menos conocida que sus contrapartes que operan con los ciclos por comprensión de vapor y absorción (Dieng y Wang, 2001).

El ciclo cerrado de tal máquina térmica parte del supuesto que el conjunto colector-generador contiene estrechamente la pareja CA-refrigerante (fluido de trabajo) y que los gases indeseados han sido purgados apropiadamente. Fig. 1 representa el ciclo ideal de un refrigerador intermitente por adsorción formado por las líneas ABDF-CE en el diagrama de Clapeyron ( $L n$ P vs-1/T). Un ciclo completo se desarrolla en dos etapas sucesivas: una de calentamiento-desorción-condensación a presión y temperatura altas (ABD) y otra de enfriamiento-adsorción-vaporización a presión y temperatura bajos (DFA). Las presiones alta y baja, Pc y PE, son de saturación del refrigerante puro correspondientes a las temperaturas del condensador y evaporador Tc y TE, respectivamente (Boubakri et al., 1992). Además, la línea CE corresponde al equilibrio o saturación liquido-vapor del refrigerante puro. Esta sucesión de etapas es tremendamente conveniente para tomar ventaja del recurso solar, el cual también alterna entre día-noche (Pons y Guilleminot, 1986).

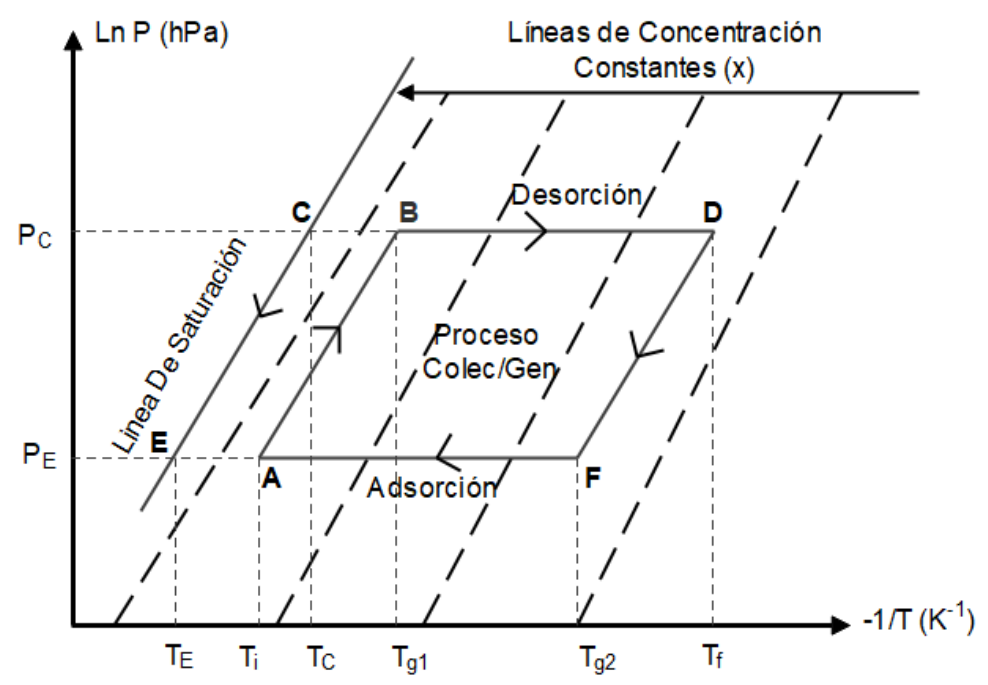

Fig.1: Diagrama (P-x-T) de Clapeyron del ciclo ideal de adsorción.

Si el ciclo inicia en el punto $A$ donde el conjunto colector-generador se encuentra en el estado de equilibrio definido por la temperatura ambiente $T_{i}$ de la madrugada y la presión baja $\mathrm{P}_{\mathrm{E}}$. El calentamiento del conjunto mediante radiación solar recibida durante la mañana sigue la trayectoria ideal $A B$, la concentración de refrigerante adsorbido es la más alta $\mathrm{x}_{\mathrm{i}}$ (proceso isocórico). Como la presión del conjunto aumentó hasta el punto crítico $\mathrm{B}$, donde el estado de equilibrio es $\mathrm{P}_{\mathrm{c}}$ y $\mathrm{T}_{\mathrm{g} 1}$, desde allí se conecta al condensador que idealmente es un foco térmico a $T_{c}$. Iniciándose el proceso de desorción (endotérmico) del refrigerante (vapor) desde el CA mientras sigue incrementado la temperatura hasta la máxima $T_{f}$ y concentración mínima $x_{f}$ en el punto $D$ (proceso isobárico). La cantidad de refrigerante puro por unidad de masa de CA que logra condensación por el intercambio de calor con el ambiente $\mathrm{a} \mathrm{T}_{\mathrm{c}}$ es la diferencia de concentraciones $\Delta \mathrm{x}=\mathrm{x}_{\mathrm{i}}-\mathrm{x}_{\mathrm{f}} \mathrm{y}$ ser transferido por gravedad al evaporador (punto C).

Lo que sigue es la trayectoria simétrica a lo largo de DFA. El enfriamiento del conjunto colector-generador es por intercambio de calor con el ambiente desde el punto $\mathrm{D}$ hasta otro punto crítico $\mathrm{F}$ cuyo estado es inferior en ambos a presión $P_{E}$ y temperatura $T_{g 2}$ (proceso isocórico). Este enfriamiento se garantiza mediante aberturas hechas al conjunto y por la puesta del sol. En este justo momento, el líquido refrigerante en el evaporador puede iniciar su vaporización, produciéndose un efecto de enfriamiento aprovechable. La adsorción (exotérmica) por parte del CA del vapor generado de refrigerante hace que retorne al colector- 
generador mientras sigue reduciendo la temperatura hasta completar un ciclo, el siguiente día en la mañana (proceso isobárico). En ésta última etapa, sucediendo durante noche-madrugada, hay posibilidad de lograr temperatura de congelación del agua almacenada en un espacio que rodea el evaporador.

La máquina que desarrolla este ciclo opera únicamente mediante el intercambio de energía en forma de calor a través de cuatro focos térmicos, ordenados así: $T_{E}<T_{i} \leq T_{C}<T_{f}$. Las parejas de adsorción convencionales operan a presiones inferiores al ambiente, lo cual requiere excelente hermeticidad para evitar la presencia de gases indeseables y el consiguiente menoscabo del desempeño de la máquina térmica (Hu, 1998). Tal máquina, al igual que sus contrapartes convencionales, logra transferir calor desde un foco frio hasta otro caliente, con la excepción de no requerir la entrada de trabajo mecánico; pero requiriendo más de dos focos térmicos. Adicionalmente, no hay necesidad de piezas móviles, ni válvulas. Otra consecuencia es que la producción de frio es intermitente o discontinua. La máquina en cuestión, como puede notarse, no es de propósitos generales sino por el contrario requiere ser diseñada y afinada para las condiciones particulares del medio adoptante (Distrito de Riohacha). El diseño a tales condiciones puede requerir preliminarmente un estudio a través del análisis termodinámico detallado basado en el primer principio, con el cual se pueda evaluar, por ejemplo, la sensibilidad del desempeño del equipo contra las variables climáticas (Qasem y Elshaarawi, 2015). Este primer análisis no tiene en cuenta los fenómenos de transporte o transferencias asociados a la masa y la energía.

El objetivo general que se persigue en el presente trabajo es analizar la sensibilidad de la operación termodinámica de un refrigerador solar (intermitente) por adsorción en términos de la eficiencia térmica (COP) con respecto a las variables climatológicas de Riohacha, mediante simulación computacional. El modelo matemático que soporta la simulación se basa fundamentalmente en los balances globales de calor total durante un ciclo, isotermas de adsorción mediante Dubinin-Astakhov, entalpia de adsorción, calores específicos y días típicos de irradiación global y temperatura ambiente. El colector-adsorbedor se asume en equilibrio térmico entre las fases: adsorbente (solido), refrigerante (vapor) y cantidad adsorbida o adsorbato (liquido). Se tuvo Especial cuidado en el tratamiento de propiedades termodinámicas de la pareja adsorbenterefrigerante extraídas de literatura de corriente principal. Esa misma literatura es clave para llevar a cabo procesos de verificación del modelo.

\section{MODELADO DEL CICLO IDEAL}

La aplicación del primer principio de la termodinámica al refrigerador solar por adsorción en términos de un balance global de calores totales durante un ciclo conduce a la igualdad de calores ganados y cedidos. Identificando los calores ganados en el colector-generador durante el calentamiento-desorción y en el evaporador (efecto de enfriamiento) durante la vaporización con los subíndices $\mathrm{T}$ y e, respectivamente y los calores cedidos en el colector-generador durante el enfriamiento-adsorción y en el condensador con los subíndices A y c, respectivamente:

$$
Q_{T}+Q_{e}-Q_{A}-Q_{C}=0
$$

Por otra parte, los balances entálpicos a cada elemento permiten determinar cada uno de los calores totales intercambiados; particularmente con los dos primeros térmicos de (1) es posible estimar el coeficiente de desempeño (COP) (Z. F. Li y Sumathy, 1999).

El balance en el colector-generador se puede partir entre los dos procesos isocórico e isobárico:

$$
Q_{T}=Q_{I S O S}+Q_{D E S}
$$

La ganancia de calor para llevar a cabo el proceso isocórico por unidad de masa de CA:

$$
\frac{\mathrm{Q}_{\mathrm{ISOS}}}{\mathrm{m}_{\mathrm{a}}}=\left(\mathrm{C} \mathrm{p}_{\mathrm{a}}+\mathrm{C} \mathrm{p}_{\mathrm{r}} \cdot \mathrm{x}_{\mathrm{i}}\right)\left(\mathrm{T}_{\mathrm{g} 1}-\mathrm{T}_{\mathrm{i}}\right)
$$

La ganancia de calor para llevar a cabo el proceso de desorción isobárico por unidad de masa de CA:

$$
\frac{Q_{D E S}}{m_{a}}=\left[C p_{a}+C p_{r} \frac{\left(x_{i}+x_{f}\right)}{2}\right]\left(T_{f}-T_{g 1}\right)+\left(x_{i}-x_{f}\right) H_{a d s}
$$

El balance en el evaporador cuantifica el efecto de enfriamiento: 


$$
\frac{Q_{e}}{m_{a}}=\left[L-C p_{r}\left(T_{c}-T_{E}\right)\right] m_{r, g} / m_{a}
$$

La cantidad de refrigerante generado que circula en el evaporador:

$$
m_{r, g} / m_{a}=\left(x_{i}-x_{f}\right)
$$

Por lo tanto, la eficacia más alta posible del ciclo en términos del coeficiente de desempeño:

$$
\mathrm{COP}=\frac{\mathrm{Q}_{\mathrm{e}} / \mathrm{m}_{\mathrm{a}}}{\mathrm{Q}_{\mathrm{T}} / \mathrm{m}_{\mathrm{a}}}
$$

La energía solar incidente requerida para un ciclo en términos de la eficiencia diaria del colector y la razón de masa de adsorbente por unidad de área de colector:

$$
\mathrm{I}_{\mathrm{G}, \min }=\frac{\left(\mathrm{Q}_{\mathrm{T}} / \mathrm{m}_{\mathrm{a}}\right)}{\eta_{\mathrm{c}}}\left(\frac{\mathrm{m}_{\mathrm{a}}}{\mathrm{A}_{\mathrm{C}}}\right)
$$

Las relaciones auxiliares para complementar los balances se relacionan con propiedades termo-físicas del adsorbente y el refrigerante e isotermas de adsorción. En el primer caso se usarán propiedades constantes e isotrópicas, independientes de la temperatura: calores específicos para CA sólido y refrigerante líquido y entalpia de vaporización del refrigerante. Para la presión de saturación del refrigerante es aplicable la relación univariante (NIST, 2016):

$$
\log P_{\text {sat }}=f\left(T_{\text {sat }}\right)=A-\frac{B}{T_{\text {sat }}+C}
$$

Para las isotermas de adsorción es aplicable una bien conocida relación bivariante de Dubinin-Astakhov (Hassan, Mohamad, Alyousef, y Al-Ansary, 2015) y el calor (entalpia) de adsorción puede asumir un valor constante $\mathrm{H}_{\mathrm{ads}}$ :

$$
x(T, P)=x_{0} \cdot \exp \left[-D\left(T \cdot \ln \left(P_{\text {sat }}(T) / P\right)\right)^{n}\right]
$$

En este caso encontrar el valor de la temperatura $T_{g 1}$ en el primer punto crítico requiere resolver la ecuación no lineal de la forma:

$$
x_{i}\left(T_{i}, P_{E}\left(T_{E}\right)\right)-x\left(T_{g 1}, P_{c}\left(T_{c}\right)\right)=0
$$

La búsqueda con un método tipo Newton-Raphson puede iniciarse en un valor igual a temperatura máxima del ciclo y especificar un criterio de paro $(\varepsilon)$. Se llama la atención en evitar el llamado indiscriminado del método numérico, esta temperatura desconocida $T_{g_{1}}$, una vez fijada la pareja, depende de tres focos térmicos y no depende de temperatura final del ciclo ni de la otra temperatura en el segundo punto crítico. El modelo matemático para la operación en equilibrio termodinámico durante un ciclo lo forma el conjunto de ecuaciones (1)-(11). Sin embargo, para el objetivo propuesto centrado en el desempeño solo hacen falta las ecuaciones (2)-(11) a ser organizadas para evaluarlas secuencialmente teniendo en cuenta:

Entradas: $T_{i}, T_{E}, T_{c}, T_{f}, m_{a} / A_{c}, \eta_{c}, \varepsilon$

Parámetros: $\mathrm{Cp}_{\mathrm{a}}, \mathrm{Cp}$, a, b, c, $\mathrm{x}_{\mathrm{o}}, \mathrm{D}, \mathrm{n}, \mathrm{H}_{\mathrm{ads}} \mathrm{L}$ (Los fijan la pareja);

Salidas: $Q_{e} / m_{a}, C O P, I_{G, m i n}$.

\section{RESULTADOS Y DISCUSIÓN}

La simulación incluyo la correlación y predicción de varios parámetros del ciclo, teniendo en cuenta en el proceso el contraste y verificación de los resultados (Camargo y Quintero, 2017), en particular las constantes utilizadas en el código que son propias de la pareja de adsorción-desorción fueron obtenidas de investigaciones experimentales, datos que se registran en la tabla1.

\section{Límites para la temperatura máxima del ciclo}

El primer resultado del modelo es la figura 2, que contiene dos gráficos, en los que se evalúa con respecto a la temperatura máxima final, el COP (figura 2a) y calor útil de enfriamiento (figura 2b), identificando los límites 
de temperatura máxima final para efectos de lograr la refrigeración en el equipo. Una observación detallada de la gráfica de la figura $2 a\left(T_{f}\right.$ vs COP) muestra que por debajo de $73.5^{\circ} \mathrm{C}$ no se refrigera y que por el contrario se añade calor a la cámara de refrigeración, evidente en un COP negativo, lo que sugiere una temperatura mínima para el ciclo, debajo de la cual no es posible funcionar como equipo frigorífico. La gráfica de la figura $2 b\left(T_{f} V_{s} Q_{e}\right)$, registra el incremento del calor útil de enfriamiento a partir del punto crítico, al comparar los dos gráficos de la figura 2, se marca una tendencia alrededor de $80{ }^{\circ} \mathrm{C}$, donde el COP se estabiliza y posteriormente tiende a decrecer mientras que el $Q_{e}$ continua creciendo casi que linealmente, esto debido a que remitiéndonos a la ecuación (1), QT empieza a crecer en mayor medida que $Q_{\mathrm{e}}$, dejando claro que la premura de un colector de alta eficiencia no es trascendental en el COP del equipo.

Tabla 1: Parámetros y variables operativas aplicadas en el modelo

\begin{tabular}{|c|c|c|c|c|}
\hline Símbolos & Parámetros & Valor & Unidades & Referencia \\
\hline Cpr & Calor especifico del refrigerante & 2.83 & $\mathrm{~kJ} / \mathrm{kg} \mathrm{K}$ & \multirow[t]{5}{*}{ (NIST, 2016) } \\
\hline$A$ & Constante del metanol & 5.20 & $\mathrm{Ln} \mathrm{hPa}$ & \\
\hline $\mathrm{B}$ & Constante del metanol & 1581.34 & & \\
\hline C & Constante del metanol & -33.50 & $\mathrm{~K}$ & \\
\hline $\mathrm{L}$ & Calor latente de vaporización & 1100.03 & $\mathrm{~kJ} / \mathrm{kg}$ & \\
\hline Сpa & Calor especifico del adsorbente & 0.93 & $\mathrm{~kJ} / \mathrm{kg} \mathrm{K}$ & \multirow{4}{*}{$\begin{array}{l}\text { (Qasem y El-Shaarawi, 2013) } \\
\text { (Wu et al., 2015) }\end{array}$} \\
\hline$x_{0}$ & Capacidad de adsorción & 0.49 & $\mathrm{~kg} / \mathrm{kg}$ & \\
\hline $\mathrm{D}$ & Constante en D-A ecuación & $2.8 \times 10^{-5}$ & $\mathrm{~K}^{-2}$ & \\
\hline $\mathrm{n}$ & Índice constante en D-A ecuación & 1.65 & & \\
\hline Hads & Entalpia de adsorción & 1400 & $\mathrm{~kJ} / \mathrm{kg}$ & (Tashtoush et al., 2010) \\
\hline$\eta_{c}$ & Eficiencia del colector & 0.4 & & \multirow[t]{2}{*}{ (Camargo y Quintero, 2017) } \\
\hline$m_{a} / A_{c}$ & $\begin{array}{l}\text { Relación masa de CA a área de } \\
\text { colector solar }\end{array}$ & 20 & $\mathrm{~kg} / \mathrm{m}^{2}$ & \\
\hline
\end{tabular}
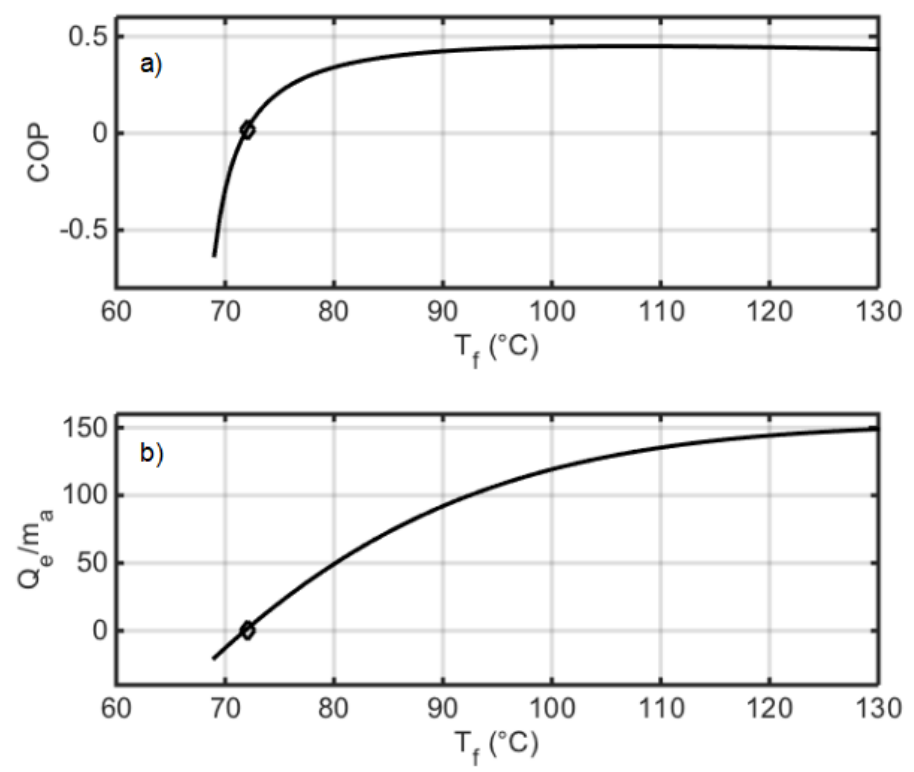

Fig. 2: $\operatorname{COP}$ (a) // Calor útil de enfriamiento (b) Vs. Temperatura máxima del ciclo

\section{Efectos de las temperaturas máximas y condensador}

En las figuras 3 y 4 , se referencia el efecto de la temperatura de condensador en el COP y el $Q_{e}$, respectivamente, la línea continua que hace referencia a $T_{c}=30^{\circ} \mathrm{C}$ tiene un mayor valor comparado con las curvas de Tc=35 y Tc=40, fenómeno que se nota tanto en el COP como en el $Q_{e}$, dando una relevancia fundamental al hecho de procurar que las temperaturas en este dispositivo sean lo más bajas posible. El punto crucial de estas gráficas es corroborar el hecho que una baja temperatura en el condensador no solamente proporciona un COP más alto, si no que incrementa el calor útil, convirtiéndose en un elemento sobre el que recae indirectamente la generación del calor del efecto de enfriamiento, por tanto al igual que en los sistemas convencionales de refrigeración requiere ser optimizado durante la etapa del diseño. 


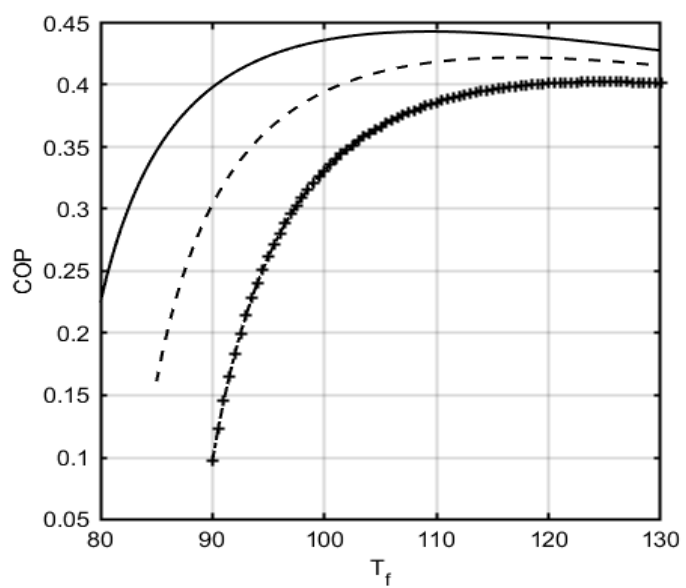

Fig. 3: COP en función de la $T_{f}$ y Condensador: $T_{c}=30(-)$, $\mathrm{T}_{\mathrm{C}}=35(--), \mathrm{T}_{\mathrm{C}}=40(-+)$.

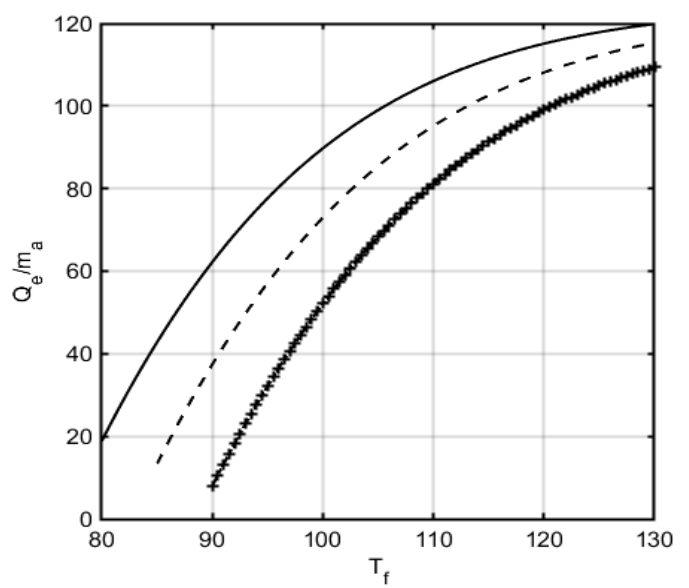

Fig. 4: Calor útil de enfriamiento en función de la $T_{f}$ y Condensador: $\mathrm{T}_{\mathrm{C}}=30(-), \mathrm{T}_{\mathrm{c}}=35(--), \mathrm{T}_{\mathrm{c}}=40(-+)$

\section{Desempeño e irradiación para un ciclo vs. Temperaturas}

Para establecer el desempeño del equipo de refrigeración por adsorción con las condiciones meteorológicas del municipio de Riohacha, se hizo un promedio de la temperatura e irradiación solar registradas a partir de los datos entregados por Meteonorm (Meteonorm software, 2016), tabulados cada hora durante un día típico por mes en el año 2013. Desde este punto, se pudo introducir los parámetros $T_{i}$ y $T_{c}$ con lo cual, se corrió el programa para obtener los resultados presentados en la tabla 2. Se aclara que los demás datos de entrada en esta tabla, fueron extraídos de una detallada revisión bibliográfica de artículos Q1, esto debido al interés de solo evaluar el comportamiento bajo las condiciones de temperatura e irradiación de Riohacha.

Tabla 2: Resultados de la simulacion con la climatologia de Riohacha

\begin{tabular}{|c|c|c|c|c|c|c|c|c|c|}
\hline & \multicolumn{9}{|c|}{ Entradas } \\
\hline & \multicolumn{2}{|c|}{$T_{e}\left({ }^{\circ} \mathrm{C}\right)=-5$} & \multicolumn{2}{|c|}{$m_{a} / A_{c}=20$} & \multicolumn{2}{|c|}{$E f i c=0,4$} & & & \\
\hline & \multicolumn{9}{|c|}{ Salidas } \\
\hline \multirow{2}{*}{ Mes } & \multirow{2}{*}{$\mathrm{T}_{\mathrm{i}}\left({ }^{\circ} \mathrm{C}\right)$} & \multirow{2}{*}{$\mathrm{T}_{\mathrm{c}}\left({ }^{\circ} \mathrm{C}\right)$} & \multirow{2}{*}{$\mathrm{T}_{\mathrm{f}}\left({ }^{\circ} \mathrm{C}\right)$} & \multirow{2}{*}{ COP } & \multicolumn{2}{|c|}{$\mathrm{Q}_{\mathrm{e}} /$ Ciclo } & \multirow{2}{*}{$\begin{array}{l}\mathrm{I}_{\mathrm{G}, \min } \\
\left(\mathrm{kWh} / \mathrm{m}^{2} / \mathrm{dia}\right)\end{array}$} & \multirow{2}{*}{$\begin{array}{l}\text { IG,real } \\
\left(\mathrm{kWh} / \mathrm{m}^{2} / \mathrm{dia}\right)\end{array}$} & \multirow{2}{*}{$\begin{array}{l}\mathrm{I}_{\mathrm{G}, \text { real }} / \mathrm{I}_{\mathrm{G}, \min } \\
\left(\mathrm{kWh} / \mathrm{m}^{2} / \text { dia }\right)\end{array}$} \\
\hline & & & & & $(\mathrm{Kj} / \mathrm{kg})$ & $\left(\mathrm{kWh} / \mathrm{m}^{2}\right)$ & & & \\
\hline \multirow[t]{3}{*}{ Ago. } & 21.9 & 27.64 & 85 & 0.52 & 183.14 & 1.02 & 4.88 & 6.85 & 1.40 \\
\hline & & & 95 & 0.53 & 212.97 & 1.18 & 5.62 & & 1.22 \\
\hline & & & 105 & 0.52 & 230.65 & 1.28 & 6.13 & & 1.12 \\
\hline \multirow[t]{3}{*}{ Sep. } & 19.6 & 26.44 & 85 & 0.53 & 210.03 & 1.17 & 5.46 & 7.81 & 1.43 \\
\hline & & & 95 & 0.54 & 238.26 & 1.32 & 6.17 & & 1.27 \\
\hline & & & 105 & 0.53 & 254.81 & 1.42 & 6.66 & & 1.17 \\
\hline \multirow[t]{3}{*}{ Oct. } & 24.6 & 27.59 & 85 & 0.51 & 152.02 & 0.84 & 4.16 & 2.92 & 0.70 \\
\hline & & & 95 & 0.52 & 181.85 & 1.01 & 4.90 & & 0.60 \\
\hline & & & 105 & 0.51 & 199.54 & 1.11 & 5.41 & & 0.54 \\
\hline \multirow[t]{3}{*}{ Nov. } & 21.2 & 26.3 & 85 & 0.53 & 188.20 & 1.05 & 4.96 & 4.73 & 0.96 \\
\hline & & & 95 & 0.53 & 216.42 & 1.20 & 5.67 & & 0.84 \\
\hline & & & 105 & 0.53 & 232.98 & 1.29 & 6.16 & & 0.77 \\
\hline \multirow[t]{3}{*}{ Dic. } & 21.6 & 27.14 & 85 & 0.52 & 183.14 & 1.02 & 4.88 & 5.30 & 1.09 \\
\hline & & & 95 & 0.53 & 212.97 & 1.18 & 5.62 & & 0.94 \\
\hline & & & 105 & 0.52 & 230.65 & 1.28 & 6.13 & & 0.86 \\
\hline
\end{tabular}

La importancia de la tabla 2 que solo es un fragmento, ya que para la tabla completa remítase a (Camargo y Quintero, 2017) muestra los resultados de la simulación y es la base para elaborar las figuras 5, 6 y 7, por lo cual es necesario explicar el contenido de ella. La parte superior de la tabla hace referencia a los parámetros de entrada suministrados al código implementado en MATLAB (Matlab R2016a, 2016), y son aspectos del diseño del equipo, la primera columna detalla el mes de donde se toman los datos de un día típico, con lo cual se hace la iteración para obtener las demás cifras del resto de la tabla. En la columna 4, que representa la temperatura máxima final $\left(\mathrm{T}_{\mathrm{f}}\right)$ se tienen tres cifras de temperatura, que son importantes pues en ese intervalo se obtiene un mayor COP, motivando a evaluar cómo se comportan los demás parámetros en tales condiciones. Las columnas de $\mathrm{T}_{\mathrm{i}}$ y $\mathrm{T}_{\mathrm{c}}$ son temperaturas ya explicadas con anterioridad. 
La columna 6 y 7 representa el calor útil del ciclo, en el caso de la columna 7 tiene unidades de $\mathrm{kWh} / \mathrm{m}^{2}$ como resultado de cálculos con los parámetros presentes en el diseño del colector, en el que se relaciona la eficiencia, la irradiación recibida y el área de captación, hay que tener en cuenta que a pesar de que las dos columnas tienen unidades diferentes ambas se refieren al calor útil, la necesidad de la conversión en la columna 7 es con el fin de operar con la ecuación (8) y obtener la irradiación global mínima (la,min). La columna final es un cociente entre la irradiación real $\left(I_{G}\right.$,real $)$ y la irradiación mínima $\left(I_{G}\right.$,min $)$ requerida para producir hielo, indicando que un valor igual o superior a 1 es certeza de producir hielo y que por debajo de este valor el equipo es para otra aplicación.

\section{Gráficas comparativas de $I_{G \text {,real }}$ e $I_{G, \min }$}

En los gráficos de la figura 5, 6 y 7 se refleja el comportamiento de la $I_{G, \text { real }}$ e $I_{G, \text { min }}$ durante un año, constatando 9 meses en los cuales el equipo con las condiciones establecidas en esta investigación puede producir hielo, arrojando una utilidad práctica del $75 \%$. Cada gráfico fue elaborado con $\mathrm{T}_{\mathrm{f}}$ de 85,95 y $105^{\circ} \mathrm{C}$, dejando evidente la simulación las condiciones favorables en cuanto a irradiación.

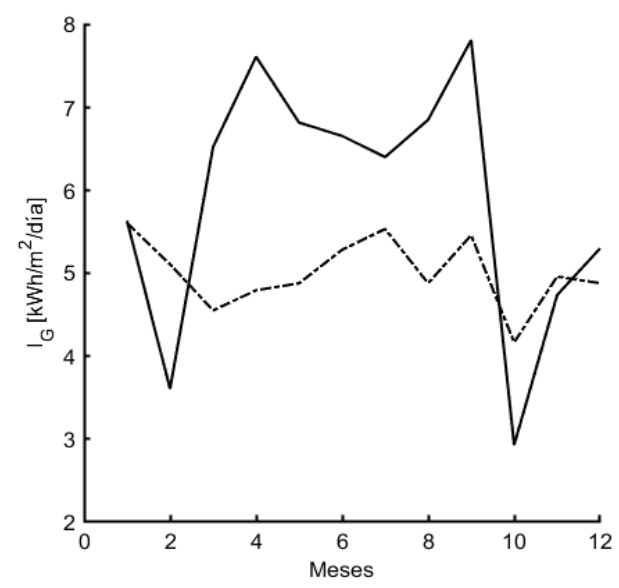

Fig. 5: Gráfica comparativa ( $\mathrm{I}_{\mathrm{G}, \mathrm{min}}$ - e $\mathrm{I}_{\mathrm{G}, \text { real }}$ - -) de un ciclo a $\mathrm{T}_{\mathrm{f}}=85^{\circ} \mathrm{C}$

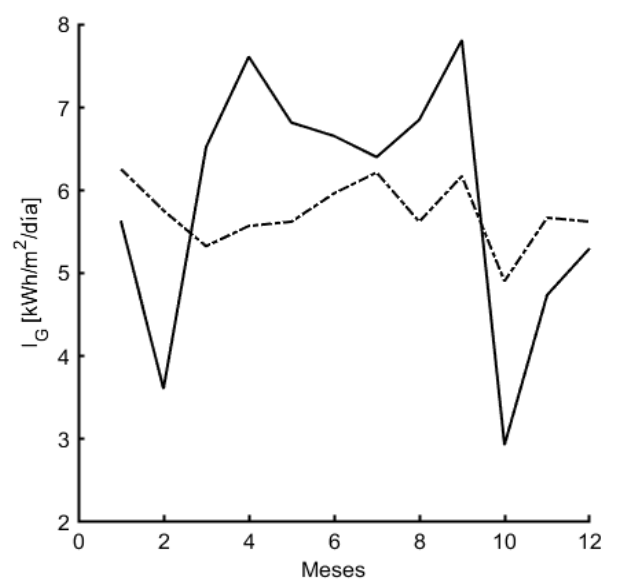

Fig. 6: Gráfica comparativa ( $\mathrm{I}_{\mathrm{G}, \min }$ - e $\mathrm{I}_{\mathrm{G}, \text { real }}$ - -) de un ciclo a $\mathrm{T}_{f}=95^{\circ} \mathrm{C}$

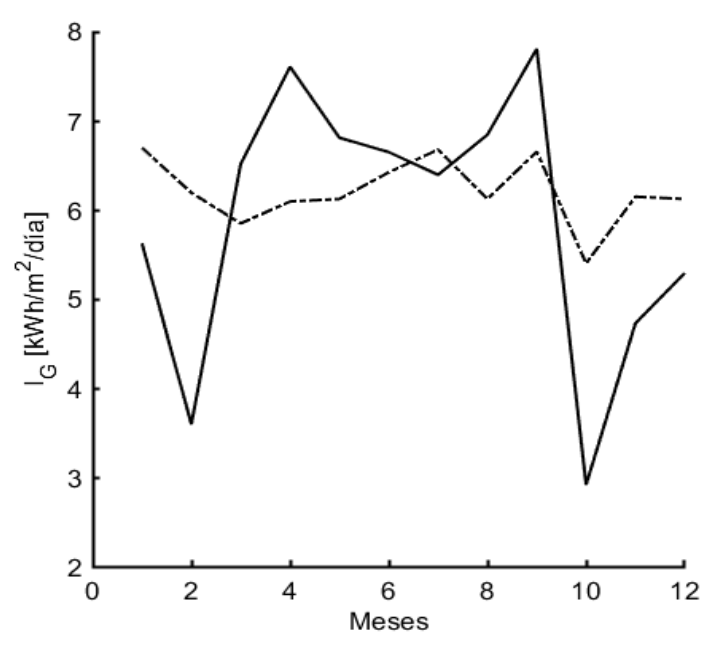

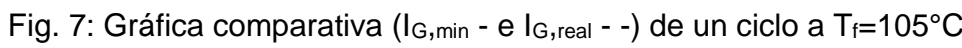

\section{Perspectivas de la investigación}

Dado que el modelo desarrollado es en estado estacionario, es útil un estudio en estado transitorio, desarrollando un análisis de segunda ley que permita el diseño de un prototipo como parte complementaria de la investigación, reiterando que los avances realizados con este trabajo entrega resultados con parámetros similares en el COP y las isosteras de adsorción a investigaciones previas con condiciones parecidas (El-Sharkawy et al., 2008). Finalmente sería importante estudiar las variantes y eficiencias del lecho adsorbente y condensador, en el que algunos autores le han imprimido aspectos novedosos (M. Li, Sun, Wang y Cai, 2004) que buscan incrementar el rendimiento de estos componentes debido al papel fundamental que tienen en el COP del refrigerador. 


\section{CONCLUSIONES}

El modelo planteado evidencia la existencia de una temperatura mínima dentro de los valores que puede tener la temperatura máxima final, indicando como por debajo de esa temperatura mínima, el equipo no cumple la función de refrigerar y, por el contrario, añade calor a la cámara del evaporador.

El análisis de sensibilidad indica que una menor temperatura de condensador y $T_{f}$ en el rango de 100 y 110 ${ }^{\circ} \mathrm{C}$ incrementa el COP, fuera de este rango el desempeño disminuye aunque $T_{c}$ sea baja, esto se desprende de la ecuación (5) y (7). Dado esto los valores de $T_{c}$ y $T_{f}$ son críticos en el diseño de los componentes para el proceso de fabricación del equipo.

La radiación y la temperatura promedio en Riohacha son favorables para el desempeño de un equipo de refrigeración por adsorción, alcanzando COP promedio de 0.52 a lo largo de un año, considerablemente, alto para este tipo de equipos. Los meses: octubre, noviembre y diciembre no tienen las condiciones de irradiación apropiadas para la producción de hielo, mientras que los meses restantes lucen óptimos para el desempeño requerido, desde este punto de vista el modelo desarrollado se puede replicar en otras localidades.

\section{AGRADECIMIENTOS}

Los autores agradecen a la Universidad de la Guajira por el apoyo brindado, especialmente al vicerrector de investigaciones Víctor Miguel Pinedo Guerra y al profesor Marlon Bastidas Barranco.

\section{REFERENCIAS.}

Boubakri, A., M. Arsalane y otros cinco autores, Experimental Study of Adsorptive Solar Powered Ice Makers in Agadir- 1, Performance in actual site, Renewable Energy, 2(1), 7-13 (1992)

Camargo, A. y J. Quintero, Simulación Computacional de la Operación del Refrigerador por Adsorción en Condiciones Climáticas de Riohacha, La Guajira, Uniguajira (2017)

Dieng, A. O. y R. Z. Wang, Literature Review on Solar Adsorption Technologies for Ice-Making and Air-Conditioning Purposes and Recent Developments in Solar Technology, Renewable and Sustainable Energy Reviews, 5(4), 313-342 (2001)

El-Sharkawy, I. I., B.B. Saha y otros cuatro autores, Experimental Investigation on Activated Carbon-Ethanol Pair for Solar Powered Adsorption Cooling Applications, International Journal of Refrigeration, 31(8), 1407-1413 (2008)

Hassan, H. Z., A. A Mohamad, Y. Alyousef y H. A. Al-Ansary, A Review on the Equations of State for the Working Pairs Used in Adsorption Cooling Systems, Renewable and Sustainable Energy Reviews, 45, 600-609 (2015)

Hu, E.J., A Study of Thermal Decomposition of Methanol in Solar Powered Adsorption Refrigeration Systems, Solar Energy, 62(5), 325-329 (1998)

IDEAM, Atlas de Radiación Solar, Ultravioleta y Ozono de Colombia, Torres Omar (2015)

Li, M., C. J. Sun, R. Z. Wang y W. D. Cai, Development of no Valve Solar Ice Maker, Applied Thermal Engineering, 24(56), 865-872 (2004)

Li, Z.F. y K. Sumathy, A Solar-Powered Ice-Maker with the Solid Adsorption Pair of Activated Carbon and Methanol, International Journal of Energy Research, 23, 517-527 (1999)

MATLAB Software, R2016a, Version 9.0 (2016)

Meteonorm Software (2016)

NIST, National Institute of Standards and Technology, Methyl Alcohol (2016)

Pons, M. y J.J. Guilleminot, Design of an Experimental Solar-Powered, Solid-Adsorption Ice Maker, Journal of Solar Energy Engineering, 108(4), 332-337 (1986)

Qasem, N.A.A. y M.A.I. El-shaarawi, Thermal Analysis and Modeling Study of an Activated Carbon Solar Adsorption Icemaker: Dhahran case study, Energy Conversion and Management, 100(August), 310-323 (2015)

Qasem, N.A. A. y M.A.I., El-shaarawi, Improving Ice Productivity and Performance for an Activated Carbon/Methanol Solar Adsorption Ice-Maker, Solar Energy, 98, 523-542 (2013)

Santori, G., S. Santamaria y otros tres autores, A Stand-Alone Solar Adsorption Refrigerator for Humanitarian Aid, Solar Energy, 100, 172-178 (2014)

Tashtoush, G.M., M. Jaradat y S. Al-Bader, Thermal Design of Parabolic Solar Concentrator Adsorption Refrigeration System, Applied Solar Energy, 46(3), 212-223 (2010)

Wu, J. W., S. H. Madani y otros cuatro autores, Characterizations of Activated Carbon-Methanol Adsorption Pair Including the Heat of Adsorptions, Journal of Chemical and Engineering Data, 60(6), 1727-1731 (2015) 